\title{
Using an emittance exchanger as a bunch compressor
}

\author{
Bruce E. Carlsten, Kip A. Bishofberger, Steven J. Russell, and Nikolai A. Yampolsky \\ Los Alamos National Laboratory, Los Alamos, New Mexico 87545, USA
}

(Received 21 February 2011; published 29 August 2011)

\begin{abstract}
A general architecture of an emittance exchanger (EEX) is considered, where the horizontal and longitudinal phase spaces are exchanged. A family of designs is described which can lead to extremely short final longitudinal lengths, even subfemtosecond. Using higher-order particle simulations, a preferred configuration is found, which has better compression capability and less emittance growth than the standard EEX design at high beam energy. An alternative design is also found which eliminates any final energy-phase coupling. Features of compression using an EEX are significantly different than with a chicane because the final longitudinal phase space is decoupled from the initial longitudinal phase space. Advantages of using an EEX for compression include less susceptibility to the coherent synchrotron radiation (CSR) microbunch instability, less susceptibility to bunch length broadening from CSR effects, and elimination of the initial energy-phase correlation that is needed for compression using a chicane as well as any residual energy-phase correlation after compression. A key disadvantage of using an EEX is that the final horizontal emittance tends to strongly depend on the initial bunch length and beam energy.
\end{abstract}

DOI: 10.1103/PhysRevSTAB.14.084403

PACS numbers: 41.85. $-\mathrm{p}, 41.60 . \mathrm{Cr}, 29.27 .-\mathrm{a}$

\section{INTRODUCTION}

The emittance exchanger (EEX) is a remarkable example of the conservation of eigenemittances [1]. Through it, a beam's longitudinal emittance is swapped with one of its transverse emittances (which, from this point on, we assume is the horizontal dimension). The EEX process was first proposed in [2] by inserting a transversely deflecting rf cavity in the middle of a chicane, to generate transverse-longitudinal coupling. In 2006, it was realized that the exchange can be made exact (in a linear sense) if the transversely deflecting rf cavity is between two doglegs with the same orientation, for a thin rf cavity $[3,4]$. The EEX was first experimentally demonstrated in 2009 [5]. Recently, a train of microbunches was produced by passing the beam through a multislit mask in front of the EEX, which forms an initial transverse beam modulation [6]. Generating more general final current distributions with an EEX has been described in [7]. All this work employed the form of the EEX described in [4] which includes two standard doglegs in the same orientation. It was recognized in [4] that second-order dispersion can lead to growth of the final horizontal emittance, and that can be minimized by employing an initial energy chirp to the beam (which is also used to minimize the effect of the thickness of the rf cavity).

The EEX has a unique ability among standard beam optics elements to customize beam parameters and the ability to generate large transformer ratios for plasma-wakefield

Published by the American Physical Society under the terms of the Creative Commons Attribution 3.0 License. Further distribution of this work must maintain attribution to the author(s) and the published article's title, journal citation, and DOI. accelerators $[7,8]$. This ability encourages one to also consider if an EEX can be used for bunch compression and it is the purpose of this paper to provide a preliminary investigation into this question. We analyze general EEX configurations, and identify specific EEX architectures that look promising. The EEX geometry is very complex when compared to a chicane, often used for subpicosecond bunch compression $[9,10]$, such as at the SLAC Linear Coherent Light Source x-ray free-electron laser [11]. Chicanes have a simple geometry consisting of dipoles with parallel pole faces to minimize geometrical emittance growth. The transfer matrix from an ideal chicane looks like a drift in the horizontal dimension with only time dispersion added (and with vertical focusing, which we will ignore throughout this paper). Bunch compression results from having an initial energy slew across the bunch, with electrons at the tail of the bunch having higher energy than those at the front. The only design choices in a typical chicane are the trades between bend angle and dipole width and between dipole width and drift between the dipoles. Chicane performance is limited by coherent synchrotron radiation (CSR) effects [12-14], including the microbunch instability [14] where slight initial current fluctuations can be magnified through a CSR-driven feedback mechanism.

Bunch compression in an EEX is very different. Because of the exchange between the horizontal and longitudinal phase spaces, the final compression only depends on the beam's initial transverse parameters, in a linear sense. This is a significant and very attractive difference, because (1) the final bunch length can be tailored with just transverse optics before the EEX without needing rf cavities, and (2) the final particle axial positions and energies can be made uncorrelated, making compression at higher energy 
attractive, and (3) the final bunch length is decoupled from the particle's energy and effects from CSR, including the microbunch instability, will be suppressed. An EEX has an unlimited number of families of related, but significantly different configurations, with different amounts of $x$-to- $z$ and $z$-to- $x$ compression or expansion. Also, some EEX configurations more strongly couple the final horizontal and longitudinal dimensions and result in a larger emittance growth from geometrical and CSR effects.

Conversely, EEX performance is limited by emittance growth due to second-order effects in the dipoles themselves. Specifically, transverse divergence arising from the rf cavity can be large at the position of the third dipole (especially for the head and tail of the bunch), leading to an induced normalized emittance growth scaling as

$$
\Delta \varepsilon_{\text {norm }} \propto \gamma a\left(\frac{l_{b}}{\eta}\right)^{2},
$$

where $\gamma$ is the beam's relativistic mass factor, $a$ is a characteristic scale length (like the length of the dipoles), $l_{b}$ is the electron bunch length, and $\eta$ is the dispersion of each dogleg. Although all EEX designs follow this scaling, some designs can lead to partial suppression of this effect. Since bunch compressors in typical applications (e.g. x-ray free-electron lasers) are at hundreds of $\mathrm{MeV}$ up to a $\mathrm{GeV}$, this scaling is significant. Because the dispersion is roughly linear with scale length $a$, this overall scaling indicates that the length of an EEX must scale roughly with beam energy, to keep the geometrical emittance growth reasonable, leading to potentially very long optics at $1 \mathrm{GeV}$.

The goal of this paper is to provide a general analysis of the ability of an EEX optic to compress an initially uncorrelated electron beam, and to identify its performance limits identified by Eq. (1). We identify what we consider to be preferred configurations where we have included additional optics into the design.

The main results of this paper are the following. We verify and quantify the advantages of using an EEX for compression listed above and determine the performance limits indicated by Eq. (1). We find a slightly modified EEX configuration which can be used to generate significantly smaller bunch lengths than the original configuration [4]. We identify a preferred EEX geometry that is the least susceptible to emittance growth, for the initial conditions we consider. We also identify an alternative preferred geometry that has an uncoupled final longitudinal phase space and that is more resistant to CSR effects. Compared to a compression in a nominal chicane design, we show that smaller final compressed bunch lengths are possible in the presence of CSR forces, with similar emittance growths. We also show that the microbunch instability is effectively suppressed and only results in energy banding with negligible effects on the final axial particle positions. Simulations are presented that illustrate these features. The material here is not intended to be exhaustive but rather to provide a list of EEX compressor characteristics that are novel and show promise for certain applications.

For the purposes of this paper, we consider the case of a $1-\mathrm{GeV}$ electron beam with equal initial normalized horizontal and longitudinal emittance, each $1.0 \mu \mathrm{m}$. These emittances are consistent with state-of-the-art $1 / 2$ to $1 \mathrm{nC}$ photoinjectors. We consider a nominal bunch compression from 500 to $25 \mathrm{fsec}$, with dipole and drift lengths on the order of $10 \mathrm{~m}$ and bend angles from 1 to 10 degrees. We additionally assume the beam has no initial transverse or longitudinal correlations. Also, we only consider optics in the horizontal and longitudinal directions, which leads to no loss of generality since vertical focusing can be included with no change in the horizontal or longitudinal tunes. Proper canonical variables for considering eigenemittances have been defined in [15], represented by $\vec{x}=\left[x, x^{\prime}, c \Delta t, \Delta(\beta \gamma) / \gamma_{0}\right]$, where the time deviation is relative to a reference particle (typically at the bunch's center, denoted by the subscript " 0 ") and is positive for electrons at the head of the bunch. Here, to simplify the notation

and because we consider highly relativistic beams, we use a beam vector defined by $\vec{x}=\left(x, x^{\prime}, z, \Delta \gamma / \gamma\right)$, where $x^{\prime}=d x / d z$ and $z$ refers to the axial position of each particle relative to the longitudinal center of the bunch. Finally, we consider optics that are not constrained by overall length.

\section{STANDARD EEX OPTICS}

Here we outline the basic EEX optics, shown in Fig. 1, as initially described in Ref. [4]. We use this definition to establish a baseline EEX architecture to use for comparison with the more general EEX definition in the following section.

An EEX nominally consists of two doglegs separated by a transversely deflecting rf cavity. The following development shows that this optics exchanges the horizontal and axial emittances, for a specific rf cavity strength. The $x-z$ transfer matrix for a dogleg is

$$
M_{\text {dog }}=\left(\begin{array}{cccc}
1 & L & 0 & \eta \\
0 & 1 & 0 & 0 \\
0 & \eta & 1 & \varepsilon \\
0 & 0 & 0 & 1
\end{array}\right)
$$

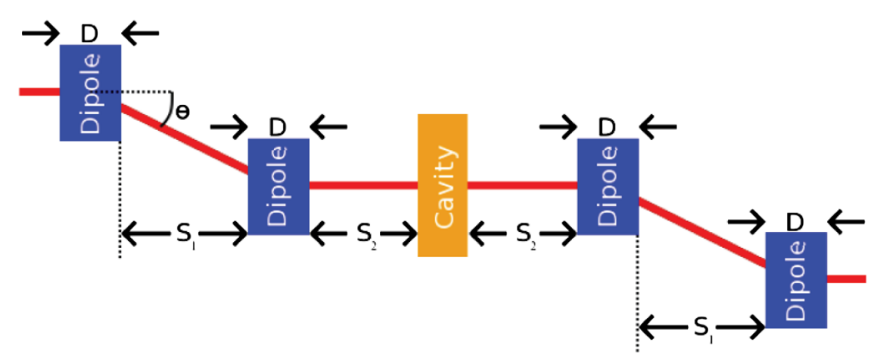

FIG. 1. Basic EEX optics. 
and the transfer matrix for an effectively thin kicker cavity is

$$
M_{\text {kicker }}=\left(\begin{array}{cccc}
1 & 0 & 0 & 0 \\
0 & 1 & k & 0 \\
0 & 0 & 1 & 0 \\
k & 0 & 0 & 1
\end{array}\right)
$$

where $k$ is the deflection voltage divided by the beam energy and rf wavelength, $k=2 \pi \mathrm{eV}_{\text {deflecting }} / \lambda \mathrm{E}$. (The linear approximation is valid when the bunch length is sufficiently shorter than the rf period, which we assume.) The combined transfer matrix of dogleg-kickerdogleg is

$$
\begin{aligned}
& M_{\text {dog }} M_{\text {kicker }} M_{\text {dog }} \\
& =\left(\begin{array}{cccc}
1+k \eta & 2 L+2 k \eta L & k L & 2 \eta+k \eta^{2}+\varepsilon k L \\
0 & 1+k \eta & k & \varepsilon k \\
\varepsilon k & 2 \eta+k \eta^{2}+\varepsilon k L & 1+k \eta & 2 \varepsilon k \eta+2 \varepsilon \\
k & k L & 0 & 1+k \eta
\end{array}\right)
\end{aligned}
$$

which turns into

$$
M_{\mathrm{dog}} M_{\mathrm{kicker}} M_{\mathrm{dog}}=\left(\begin{array}{cccc}
0 & 0 & -\frac{L}{\eta} & \eta-\frac{\varepsilon L}{\eta} \\
0 & 0 & -\frac{1}{\eta} & -\frac{\varepsilon}{\eta} \\
-\frac{\varepsilon}{\eta} & \eta-\frac{\varepsilon L}{\eta} & 0 & 0 \\
-\frac{1}{\eta} & -\frac{L}{\eta} & 0 & 0
\end{array}\right)
$$

if the kicker strength is picked such that $k \eta=-1$ [4]. If we write

$$
T=M_{\mathrm{dog}} M_{\mathrm{kicker}} M_{\mathrm{dog}}=\left(\begin{array}{cc}
0 & B \\
A & 0
\end{array}\right),
$$

an initial uncorrelated beam matrix

$$
\sigma_{\text {beam }}=\left(\begin{array}{cc}
\sigma_{x} & 0 \\
0 & \sigma_{z}
\end{array}\right)
$$

transforms into

$$
\begin{aligned}
\sigma_{\text {new }} & =\left(\begin{array}{cc}
0 & A^{T} \\
B^{T} & 0
\end{array}\right)\left(\begin{array}{cc}
\sigma_{x} & 0 \\
0 & \sigma_{z}
\end{array}\right)\left(\begin{array}{cc}
0 & B \\
A & 0
\end{array}\right) \\
& =\left(\begin{array}{cc}
A^{T} \sigma_{z} A & 0 \\
0 & B^{T} \sigma_{x} B
\end{array}\right) .
\end{aligned}
$$

Since $\operatorname{det} A=\operatorname{det} B=1$, the horizontal and longitudinal emittances are exchanged. The assumption of a thin rf cavity is not limiting. With sufficient optics, any combination of thick rf transversely deflecting cells can be made to have a transfer matrix of the form shown in Eq. (3), as shown in Appendix A. We will use the terminology that an rf cavity consists of multiple rf cells within it.

For an electron in the beam, its final coordinates given by the vector $\vec{x}_{f}$ are related to its initial coordinates $\vec{x}_{i}$ by

$$
\begin{aligned}
\left(\begin{array}{c}
x_{f} \\
x_{f}^{\prime} \\
z_{f} \\
(\Delta \gamma / \gamma)_{f}
\end{array}\right) & =\left(\begin{array}{cccc}
0 & 0 & -\frac{L}{\eta} & \eta-\frac{\varepsilon L}{\eta} \\
0 & 0 & -\frac{1}{\eta} & -\frac{\varepsilon}{\eta} \\
-\frac{\varepsilon}{\eta} & \eta-\frac{\varepsilon L}{\eta} & 0 & 0 \\
-\frac{1}{\eta} & -\frac{L}{\eta} & 0 & 0
\end{array}\right)\left(\begin{array}{c}
x_{i} \\
x_{i}^{\prime} \\
z_{i} \\
(\Delta \gamma / \gamma)_{i}
\end{array}\right) \\
& \left(\begin{array}{c}
-\frac{L}{\eta} z_{i}+\left(\eta-\frac{\varepsilon L}{\eta}\right)(\Delta \gamma / \gamma)_{i} \\
-\frac{1}{\eta} z_{i}-\frac{\varepsilon}{\eta}(\Delta \gamma / \gamma)_{i} \\
-\frac{\varepsilon}{\eta} x_{i}+\left(\eta-\frac{\varepsilon L}{\eta}\right) x_{i}^{\prime} \\
-\frac{1}{\eta} x_{i}-\frac{L}{\eta} x_{i}^{\prime}
\end{array}\right),
\end{aligned}
$$

where, for an ultrarelativistic beam,

$$
\begin{gathered}
L=S_{1} \frac{1}{\cos ^{3} \theta_{0}}+2 \frac{D}{\cos \theta_{0}}+S_{2} \\
\eta=S_{1} \frac{\sin \theta_{0}}{\cos ^{2} \theta_{0}}+2 \frac{D}{\sin \theta_{0}}\left(\frac{1}{\cos \theta_{0}}-1\right) \\
\varepsilon=S_{1} \frac{\sin ^{2} \theta_{0}}{\cos ^{3} \theta_{0}}+2 \frac{D}{\sin \theta_{0}}\left(\frac{\sin \theta_{0}}{\cos \theta_{0}}-\theta_{0}\right) .
\end{gathered}
$$

Note that the dogleg parameters $\eta$ and $\varepsilon$ define $S_{1}$ and $D$, and then the overall length $L$ defines $S_{2}$, and vice versa. It is useful to consider rough order of magnitude estimates for the deflecting cavity, to identify what field strengths are reasonable. An achievable field amplitude for an X-band transverse rf cell is $100 \mathrm{MV} / \mathrm{m}$, so a cavity with nominally $10 \mathrm{rf}$ cells with a $1-\mathrm{GeV}$ electron beam has $k=-\frac{1}{\eta} \sim 1 \mathrm{~m}^{-1}$.

Equation (6) indicates that the final beam bunch length can be made small by decreasing the horizontal beam size at the start of the EEX, to a limit arising from the initial beam divergence. For an uncorrelated initial beam, the final sizes are given by

$$
z_{f, \mathrm{rms}}=\sqrt{\left(\frac{\varepsilon}{\eta}\right)^{2}\left\langle x_{i}^{2}\right\rangle+\left(\eta-\frac{\varepsilon L}{\eta}\right)^{2}\left\langle x_{i}^{\prime 2}\right\rangle}
$$

and

$$
x_{f, \mathrm{rms}}=\sqrt{\left(\frac{L}{\eta}\right)^{2}\left\langle z_{i}^{2}\right\rangle+\left(\eta-\frac{\varepsilon L}{\eta}\right)^{2}\left\langle\left(\frac{\Delta \gamma}{\gamma}\right)^{2}\right\rangle} .
$$

Note that even for high energy beams, the term depending on $\left\langle x_{i}^{\prime 2}\right\rangle^{1 / 2}$ may not be insignificant on the final axial beam size. For a $1-\mathrm{GeV}, 1-\mu \mathrm{m}$ emittance, $100-\mu \mathrm{m}$ radius beam, $\left\langle x_{i}^{12}\right\rangle^{1 / 2} \sim 10^{-5}$ so for geometries with high dispersion or momentum compaction, this term can limit bunch length compression to $\sim 100 \mu \mathrm{m}$ ( $\sim 1 / 3 \mathrm{psec})$. This level of compression may not be adequate for some applications, and certainly not for using a transversely masked EEX to prebunch the electron beam for x-ray free-electron lasers at subnanometer wavelengths as proposed in [16,17], which would require an exact 1:1 mapping from the initial 
transverse to final axial positions, including possible magnification or reduction. Other applications may require swapping of especially small emittances or no residual energy-phase correlation in the beam.

In order to consider compression to very short bunch lengths, we will consider EEX geometries where the final axial position is only a function of a particle's initial horizontal position. Alternatively, we can consider where $z_{f}$ is only a function of initial divergence $x_{i}^{\prime}$, but since initial optics can be found to exchange $x_{i}$ and $x_{i}^{\prime}$, those cases are not unique. Since the 1:1 horizontal to axial mapping can be done in many ways, an important metric in evaluating EEX performance is susceptibility to geometrical emittance growth, as described by Eq. (1).

For the conventional EEX architecture, we can map $x_{i}$ directly to $z_{f}$ and eliminate the compression limitation from the beam's initial horizontal divergence if

$$
\eta-\frac{\varepsilon L}{\eta}=0 \quad \text { or } \quad L=\frac{\eta^{2}}{\varepsilon} .
$$

With this condition, $z_{i}$ is also directly mapped to $x_{f}$, so the final beam sizes become

$z_{f, \mathrm{rms}}=\left|\frac{\varepsilon}{\eta}\right| x_{i, \mathrm{rms}}$ and $x_{f, \mathrm{rms}}=\left|\frac{L}{\eta}\right| z_{i, \mathrm{rms}}=\left|\frac{\eta}{\varepsilon}\right| z_{i, \mathrm{rms}}$.

Note the product of the beam sizes remains constant-if the beam is compressed longitudinally, it will expand transversely, and second-order nonlinearities in dipoles can lead to an unacceptable emittance growth. It is possible to avoid this expansion by focusing the beam to a really small initial horizontal size and letting $|\varepsilon|=|\eta|$, but that may be hard to do (e.g., a final bunch length of $25 \mathrm{fsec}$ would require transverse focusing at the front of the EEX to a radius about 7 microns).

Direct evaluation of $L=\frac{\eta^{2}}{\varepsilon}$ shows that either $S_{1}$ or $S_{2}$ must be negative, which, in principle, is okay. Making $S_{2}<0$ is preferable to making $S_{1}<0$ because it is much easier to do, and its value is given by $S_{2}=\frac{\eta^{2}}{\varepsilon}-$ $\left[S_{1} /\left(\cos ^{3} \theta_{0}\right)\right]-\frac{2 D}{\cos \theta_{0}}$. The two dispersions $\eta$ in the dogleg transfer matrix shown in Eq. (1) much be equal; one comes from the dependency of the final horizontal position on initial particle energy and the other from the dependency of the final longitudinal position on initial horizontal divergence. If $S_{1}<0$, design care must be taken to ensure that both dispersion terms are equally effected. There is no such

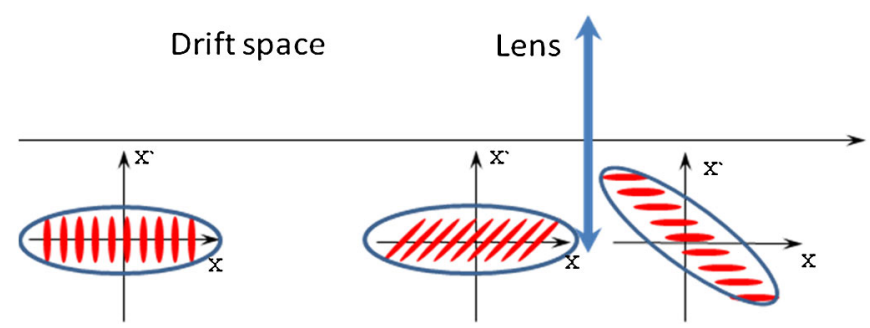

FIG. 2. Optics to mix the horizontal position and horizontal divergence to provide an additional degree of freedom in EEX design.

complication for making $S_{2}<0$, where simple optics (quadrupoles and drifts) can be used, with one possible optics option identified in Appendix II.

\section{ALTERNATIVE EEX CONFIGURATIONS}

The purpose of this section is to provide an overview of a more general EEX definition, which will provide additional control of the final beam transverse and longitudinal sizes, by generating specific horizontal correlations in the beam. The EEX can be generalized by implementing a drift followed by a lens before the EEX as shown in Fig. 2, with a transfer matrix of the form

$$
M_{\text {front }}=\left(\begin{array}{cc}
1 & a \\
b & 1+a b
\end{array}\right)
$$

which now provides an arbitrary mixing between the initial horizontal position and divergence, including even the ability to transfer an initial horizontal modulation to a modulation in divergence.

In general, this transform can provide an initial mixed transverse correlation to the EEX. It needs to be pointed out that an equivalent mixed longitudinal correlation in the initial beam can be generated either by a collection of slew rf cavities or a previous EEX optic as shown in [18]. Such a mixed longitudinal correlation has been shown to help limit the final transverse emittance growth [4]. Here, however, we limit our discussion to only mixed initial transverse distributions, to provide some bounds to the number of considered configurations and because we are primarily interested in controlling the final longitudinal phase space.

With the initial mixed transverse correlation, the final particle vector is given by

$$
\left(\begin{array}{c}
x_{f} \\
x_{f}^{\prime} \\
z_{f} \\
(\Delta \gamma / \gamma)_{f}
\end{array}\right)=\left(\begin{array}{c}
-\frac{L}{\eta} z_{i}+\left(\eta-\frac{\varepsilon L}{\eta}\right)(\Delta \gamma / \gamma)_{i} \\
-\frac{1}{\eta} z_{i}-\frac{\varepsilon}{\eta}(\Delta \gamma / \gamma)_{i} \\
{\left[-\frac{\varepsilon}{\eta}+b\left(\eta-\frac{\varepsilon L}{\eta}\right)\right] x_{i}+\left[-\frac{\varepsilon a}{\eta}+(1+a b)\left(\eta-\frac{\varepsilon L}{\eta}\right)\right] x_{i}^{\prime}} \\
\left(-\frac{1}{\eta}-\frac{L}{\eta} b\right) x_{i}+\left(-\frac{a}{\eta}-\frac{L}{\eta}(1+a b)\right) x_{i}^{\prime}
\end{array}\right)
$$


Since the axial position is given in terms of the particle positions at the start of the drift by $z_{f}=\varepsilon k\left(x_{i}+a x_{i}^{\prime}\right)+$ $(\eta+\varepsilon k L)\left[b x_{i}+(1+a b) x_{i}^{\prime}\right]$, the more generalized form of Eq. (12) for direct horizontal to axial mapping is

$$
\left(\eta-\frac{\varepsilon L}{\eta}\right)(1+a b)=\frac{\varepsilon a}{\eta} .
$$

Note if $a=0$, the condition from the previous subsection is recovered. This expression relates $a, b$, and $L$, and specifying any two will recover the third. Specifically,

$$
\begin{gathered}
a=\left(\eta-\frac{\varepsilon L}{\eta}\right) /\left[\frac{\varepsilon}{\eta}-b\left(\eta-\frac{\varepsilon L}{\eta}\right)\right] \\
b=\left(\frac{\varepsilon}{\eta}\right) /\left(\eta-\frac{\varepsilon L}{\eta}\right)-1 / a \quad(a \neq 0) \\
L=\frac{\eta}{\varepsilon}\left(\eta-\frac{\varepsilon a}{\eta(1+a b)}\right) \quad(a b \neq-1) .
\end{gathered}
$$

Using $\varepsilon=\eta^{2} /[L+a /(1+a b)]$, we can write the compressed final axial size as

$$
z_{f, \mathrm{rms}}=\left|\frac{\varepsilon}{(1+a b) \eta}\right|\left\langle x_{i}^{2}\right\rangle^{1 / 2}=\left|\frac{\eta}{(1+a b) L+a}\right|\left\langle x_{i}^{2}\right\rangle^{1 / 2} .
$$

Also note that the final transverse size is given by

$$
x_{f, \mathrm{rms}}=\sqrt{\left(\frac{L}{\eta}\right)^{2}\left\langle z_{i}^{2}\right\rangle+\left(\frac{\eta a}{L+L a b+a}\right)^{2}\left\langle\left(\frac{\Delta \gamma}{\gamma}\right)^{2}\right\rangle} .
$$

These equations are the main result of this section. They provide the scaling of the final beam sizes as functions of the initial beam sizes and EEX design parameters.

Note that the energy dependent term in Eq. (21) can be easily kept to $<100 \mu \mathrm{m}$, for energy spreads $\sim 10^{-4}$, since $\eta \sim 1 \mathrm{~m}$. There are several approaches to find values for $S_{1}, S_{2}$, and $D$ that satisfy the condition in Eq. (16) and possible compression in both dimensions simultaneously.

In addition to minimizing the compressed bunch length, it is also possible to remove any final energy-phase correlation, which may be desirable for some applications (e.g. final compression at full energy). Considering Eq. (15), there are two approaches to do this. One approach is to have $z_{f}$ only a function of initial horizontal position and $(\Delta \gamma / \gamma)_{f}$ only a function of initial horizontal divergence and the other approach is to have the opposite dependencies. If the first option is picked, the constraints are $a=\left(\eta-\frac{\varepsilon L}{\eta}\right) /\left[\frac{\varepsilon}{\eta}-b\left(\eta-\frac{\varepsilon L}{\eta}\right)\right]$ and $b L=-1$, or $a=$ $L-\left[\left(\varepsilon L^{2}\right) / \eta^{2}\right]$ and $b L=-1$, which are reasonable. Surprisingly, negative drifts are not required. For example, if $S_{1}=0$ (the dipoles are up against each other) and we ignore $S_{2}$, then $a=L-\left[\left(\varepsilon L^{2}\right) / \eta^{2}\right] \approx$ $L\left[1-\frac{\sin \theta_{0}}{\cos \theta_{0}}\left(\left\{1-\left[\left(\theta_{0} \cos \theta_{0}\right) /\left(\sin \theta_{0}\right)\right]\right\} /\left(1-\cos \theta_{0}\right)\right)\right]$ and all lengths can stay positive. The final particle vector becomes

$$
\left(\begin{array}{c}
x_{f} \\
x_{f}^{\prime} \\
z_{f} \\
(\Delta \gamma / \gamma)_{f}
\end{array}\right)=\left(\begin{array}{c}
\frac{1}{b \eta} z_{i}-b a \eta(\Delta \gamma / \gamma)_{i} \\
-\frac{1}{\eta} z_{i}+\eta b(1+a b)(\Delta \gamma / \gamma)_{i} \\
b \eta x_{i} \\
\frac{1}{b \eta} x_{i}^{\prime}
\end{array}\right)
$$

and the final longitudinal phase space is clearly decoupled with no initial horizontal correlations.

The second option is okay also, with constraints $-a=$ $L(1+a b)$ and $\frac{\varepsilon}{b \eta}=\eta-\frac{\varepsilon L}{\eta}$. The final particle vector for that case is

$$
\left(\begin{array}{c}
x_{f} \\
x_{f}^{\prime} \\
z_{f} \\
(\Delta \gamma / \gamma)_{f}
\end{array}\right)=\left(\begin{array}{c}
-\frac{L}{\eta} z_{i}-\frac{a \eta}{L}(\Delta \gamma / \gamma)_{i} \\
-\frac{1}{\eta} z_{i}+\frac{\eta a b}{L}(\Delta \gamma / \gamma)_{i} \\
-\frac{a \eta}{L} x_{i}^{\prime} \\
\frac{L}{a \eta} x_{i}
\end{array}\right) .
$$

The second option may have advantages for keeping the rf cavity field low, because we can pick lengths like $\eta \approx 100 \mathrm{~m}, L \approx 100 \mathrm{~m}$, and $a \approx 10 \mathrm{~m}$, leading to compressions of $a \eta / L \approx 10 \mathrm{~m}$.

\section{PREFERRED EEX CONFIGURATIONS FOR COMPRESSION}

The performance of four preferred EEX configurations are compared in this section, using the architectures defined in the previous two sections. Specifically, we will consider bunch compression in an EEX at a beam energy of $1 \mathrm{GeV}$, with characteristic scale lengths of $10 \mathrm{~m}$ (for the dipole widths and drifts, whenever possible). Equation (1) indicates that there will be a maximum precompressed bunch length for each design that preserves the low initial emittances. Numerical simulations presented in this section will show that emittances as low as $1 \mu \mathrm{m}$ can be preserved, for bunch lengths not exceeding the order of 1 ps.

We assume nominal EEX parameters where $D=S_{1}=$ $10 \mathrm{~m}$ from Fig. 1, with $3.5^{\circ}$ bends, which leads to $\eta=$ $-1.225 \mathrm{~m}$ and $\varepsilon=0.06241 \mathrm{~m}$. The nominal horizontal to longitudinal compression factor $|\varepsilon / \eta|$ is then 0.05095 (about a factor of 20). To end up with a 25 -fsec long bunch (7.5 microns), we start with a beam with an rms horizontal size of about $150 \mu \mathrm{m}$.

The four cases presented here have been taken from a larger study [19]. These cases were picked to illuminate certain features of EEX compression. The first case, case A, uses the conventional EEX geometry defined in Sec. II, where $S_{2}=10 \mathrm{~m}$. Minimum bunch compression in this case is limited by the initial beam transverse divergence, as indicated by Eq. (10). The second case, case B, uses the conventional EEX geometry but where $S_{2}$ is allowed to become negative in order to satisfy the condition in Eq. (12) [which removes the final bunch length dependency on initial beam transverse divergence as shown in Eq. (13)]. The third case, case C, uses the more general EEX geometry defined in Sec. III to minimize the 
transverse emittance growth. Specifically, the initial drift length $a$ and the overall length $L$ are chosen to do this, by letting $L=0$ (which minimizes the final transverse beam size). Additionally, $b$ is chosen to also vanish to simplify the optics. In [19], case $C$ was shown to have the lowest amount of transverse emittance growth of all cases studied. The fourth case, case D, uses the constraints leading to Eq. (22) where the final longitudinal phase space is uncorrelated. The four cases are summarized in the following table:

\begin{tabular}{|c|c|c|c|}
\hline Case & Description & Defining parameters & Compression \\
\hline A & Original EEX geometry & $S_{2}=10 \mathrm{~m}$ & $|\varepsilon / \eta|=0.0510$ \\
\hline B & $\begin{array}{l}\text { Near-original EEX geometry, but with a negative central drift to eliminate } \\
\text { bunch length dependence on transverse divergence }\end{array}$ & $\begin{array}{l}S_{2}=-6.049 \mathrm{~m} \\
L=24.044 \mathrm{~m}\end{array}$ & $|\varepsilon / \eta|=0.0510$ \\
\hline $\mathrm{C}$ & $\begin{array}{l}\text { Features of B, using more general EEX geometry with a leading drift } \\
\text { and lens, with optics defined to minimize emittance growth }\end{array}$ & $\begin{array}{l}b=0, L=0, \\
a=24.0439 \mathrm{~m}, \\
S_{2}=-30.0935 \mathrm{~m}\end{array}$ & $|\varepsilon / \eta|=0.0510$ \\
\hline $\mathrm{D}$ & $\begin{array}{l}\text { Features of B, using more general EEX geometry with a leading drift } \\
\text { and lens to have a final uncorrelated longitudinal phase space }\end{array}$ & $\begin{array}{l}b=-0.0399 \mathrm{~m}^{-1} \\
a=-1.0954 \mathrm{~m} \\
S_{2}=-5.000 \mathrm{~m}\end{array}$ & $|b \eta|=0.0488$ \\
\hline
\end{tabular}

Here and in the following section, we use the 3D code PUSHER [20] to evaluate the EEX performance. PUSHER is a code optimized for studying CSR effects, and solves symplectic electron motion in a magnetic field using a rigid $x$, $y$, and $z$ coordinate system. PUSHER solves particles' exact trajectory in drifts and dipoles (including every order of nonlinearity), but uses linear maps for other components (like quadrupoles and rf cavities), and assumes the dipoles have hard edges. These approximations have been validated by simulations with MARYLIE/IMPACT, which have verified that transverse emittance growth in EEX optics are strongly dominated by the nonlinearities in the dipoles themselves. Since both the EEX and chicane geometries use dipoles with all pole faces parallel, PUSHER is an ideal simulation tool, with the vertical motion ignored. To simulate CSR, the electric field in the direction of motion of the bunch is calculated using the formalism in [21], which in 1D reduces to the common Saldin expressions [22] that, for example, are used in ELEGANT [23]. (These 1D expressions are used in the CSR simulations in the following section.) PUSHER has been benchmarked in the steady-state condition with the exact analytic expressions [24,25]. For these and the following simulations, we use an initial $1-\mathrm{GeV}$ beam that is Gaussian both longitudinally and in energy deviation with a four-dimensional KapchinskyVladimirsky (K-V) transverse distribution, represented by 50000 particles. The initial normalized beam emittances are $1 \mu \mathrm{m}$ in all three dimensions, which, using the beam vector defined earlier, means that the rms product of the bunch length and energy spread is 1.71 psec-keV (e.g., a 0.5 -psec long initial bunch has an rms energy spread of $3.42 \mathrm{keV}$ ). The following simulations also use the nominal $10 \mathrm{~m}$ dipole and drift lengths with 3.5-degree dipole bend angles whenever possible. Other EEX parameters are chosen so the final bunch length is about 25 fsec.

An EEX has excellent compression behavior, as seen in Fig. 3 for cases A and B (cases C and D have equivalent compression capability to B). Using the nominal beam parameters, cases B-D are capable of compression to roughly 0.1 fsec, below which is dominated by the bunch's initial energy spread. Reduction of the initial energy spread leads to compression as short as 20 asec.

The final transverse emittance is plotted for all cases in Fig. 4, as a function of initial bunch length. In all cases, the final longitudinal emittance remains very nearly $1 \mu \mathrm{m}$, indicating the transport nonlinearities do not affect emittance exchange into that dimension. The emittance growth

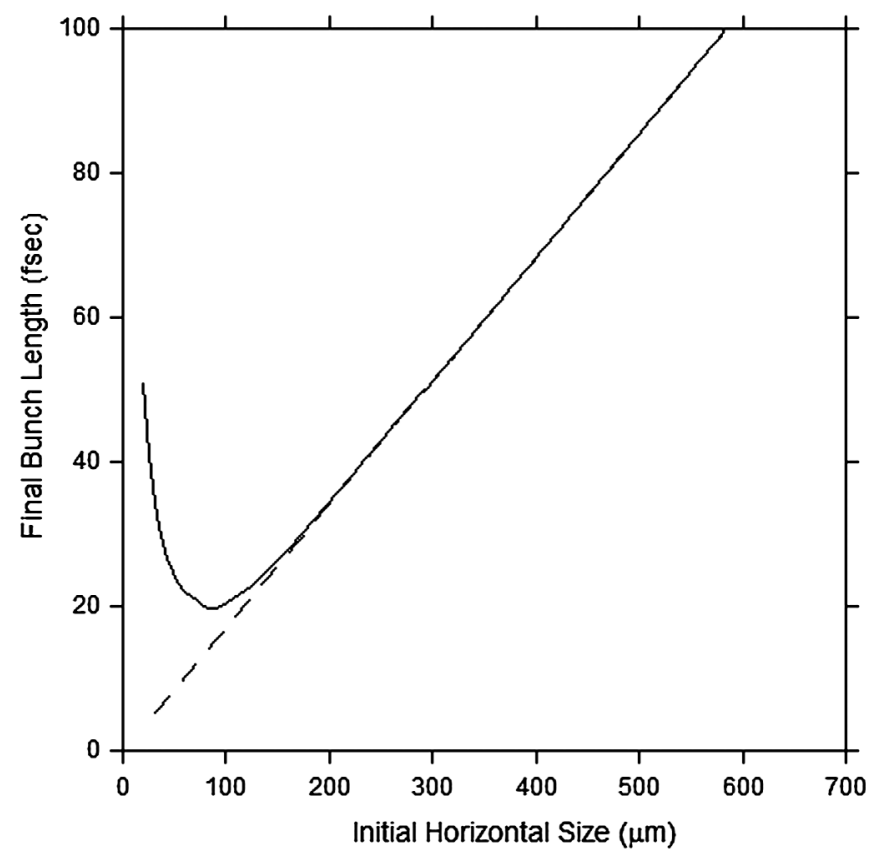

FIG. 3. Final bunch length for the nominal beam with a 0.25-ps initial bunch length as a function of initial transverse beam size for a standard EEX geometry (case A—solid line) and for case B (dashed line). 


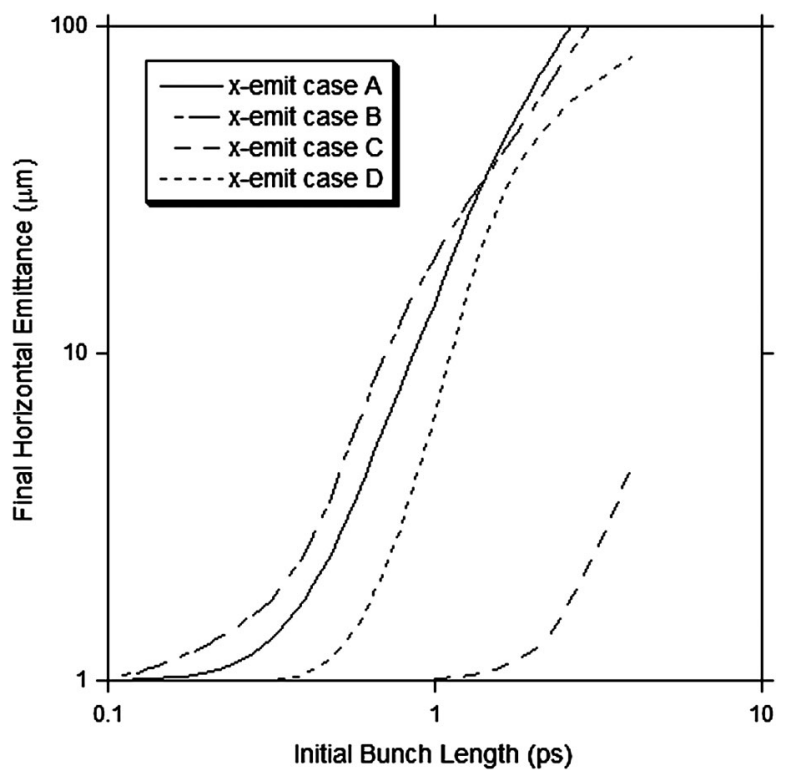

FIG. 4. Final horizontal emittance versus initial bunch length for cases A through D. Case C has the best performance, with final emittances of $1.10,1.19$, and $4.94 \mu \mathrm{m}$ for bunch lengths of 1,2 , and 4 ps, respectively. Final bunch lengths for all cases was very nearly 25 fsec.

comes from nonlinear transport through the final dipoles due to the large transverse kick from the transversely deflecting cavity, scaling as the product of the rf cavity amplitude $k$ with the longitudinal position $z$ of particles at the ends of the bunch as described by Eq. (1). Other simulations [19] show that the final transverse emittance is nearly independent of initial transverse size. This plot shows the main disadvantage of using an EEX for compression instead of a chicane. The scaling shown in Eq. (1)

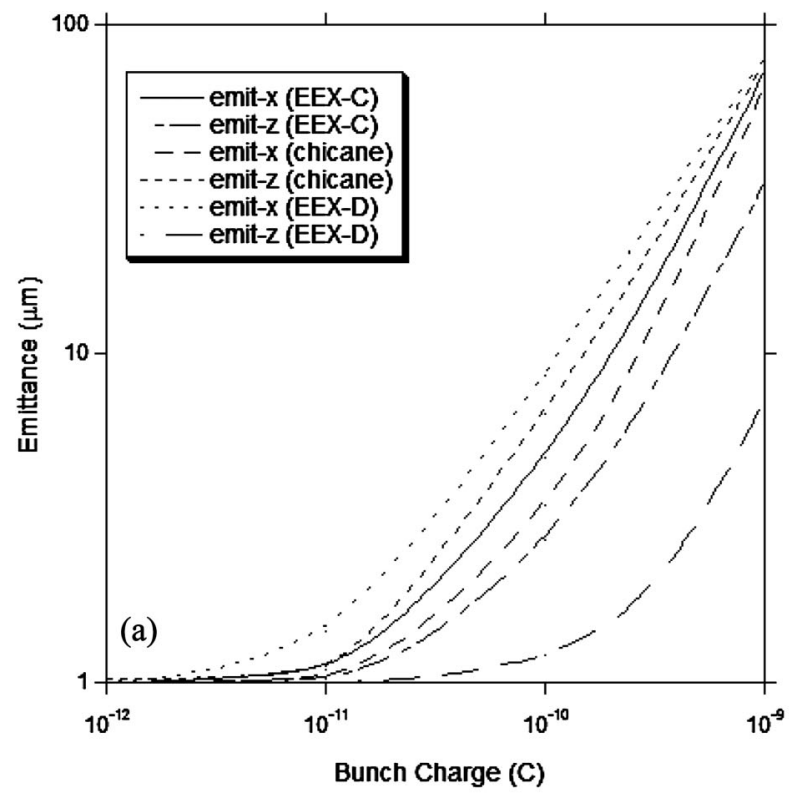

leads to a practical limitation, either in beam energy or maximum initial bunch length. It is worth noting that case $\mathrm{C}$ has about an order of magnitude improvement in bunch length tolerance over the original geometry, cases A and $\mathrm{B}$, and that making the final longitudinal phase space uncorrelated (case D) does not lead to an excessive decrease in performance.

\section{CSR DEGRADATION AND COMPARISON TO COMPRESSION IN A CHICANE}

In this final numerical section, we use PUSHER to evaluate an EEX's sensitivity to CSR effects, and compare its performance directly to an equivalent chicane, as a function of bunch charge. Of special interest is case $\mathrm{D}$, because its unique final longitudinal phase space is especially relevant to a CSR study. The momentum compaction in a chicane is given by $M_{56}=\frac{4}{3} \rho \theta^{3}$. Using 3.5-degree dipole angles, similar compressions ( 0.5 to $25 \mathrm{fsec})$ are achieved with initial energy spreads on the order of $\Delta \gamma / \gamma \approx 10^{-3}$. Specifically, using 10-m dipoles and 10-m drifts between the dipoles, compression to a nominal final bunch length of $25.7 \mathrm{fsec}$ is found with an rms energy slew of $1.14 \mathrm{MeV}$ for a $1-\mathrm{GeV}$ electron beam in such a chicane. No additional focusing has been added for the chicane, or the EEX, to account for the CSR forces. It should be pointed out that these are ridiculously high compressions-up to $40 \mathrm{kA}$ peak current for the $1 \mathrm{nC}$ case. These simulations are intended to provide a comparison between the types of effects CSR introduces in a chicane and an EEX.

In Fig. 5(a), we compare the horizontal and longitudinal emittances in both EEX designs and a chicane, for different bunch charges. All emittance growths are roughly similar

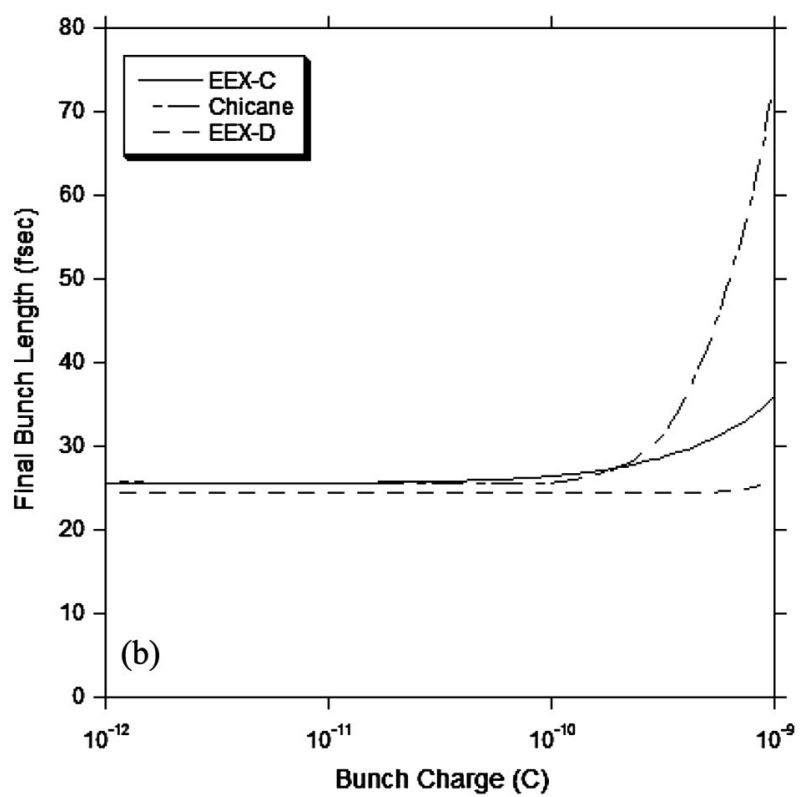

FIG. 5. (a) Horizontal and longitudinal emittance comparison between a chicane and an EEX. Emittance growths are comparable. (b) Increase in final bunch length resulting from CSR, for a chicane and an EEX. 
(including now the final longitudinal emittance), except there is less longitudinal growth for EEX case D. Note that the longitudinal emittance growth for the chicane is apparent in these simulations because of the intrinsically low base emittances, which is an order or 2 of magnitude smaller than typically considered for compression. Also note that the chicane geometry couples the CSR fields more strongly to increasing the final bunch length, shown in Fig. 5(b), due to the stronger coupling of particle energy to final axial position throughout the system. Alternatively, the increase in final bunch length is most suppressed by EEX case D because it has the highest amount of decoupling in the final longitudinal phase space. For comparison, the initial longitudinal phase space (the same for all three cases, and before the energy slew is added for the chicane) and the final longitudinal phase spaces are shown in Figs. 6(a)-6(d), for the cases of negligible bunch charge.
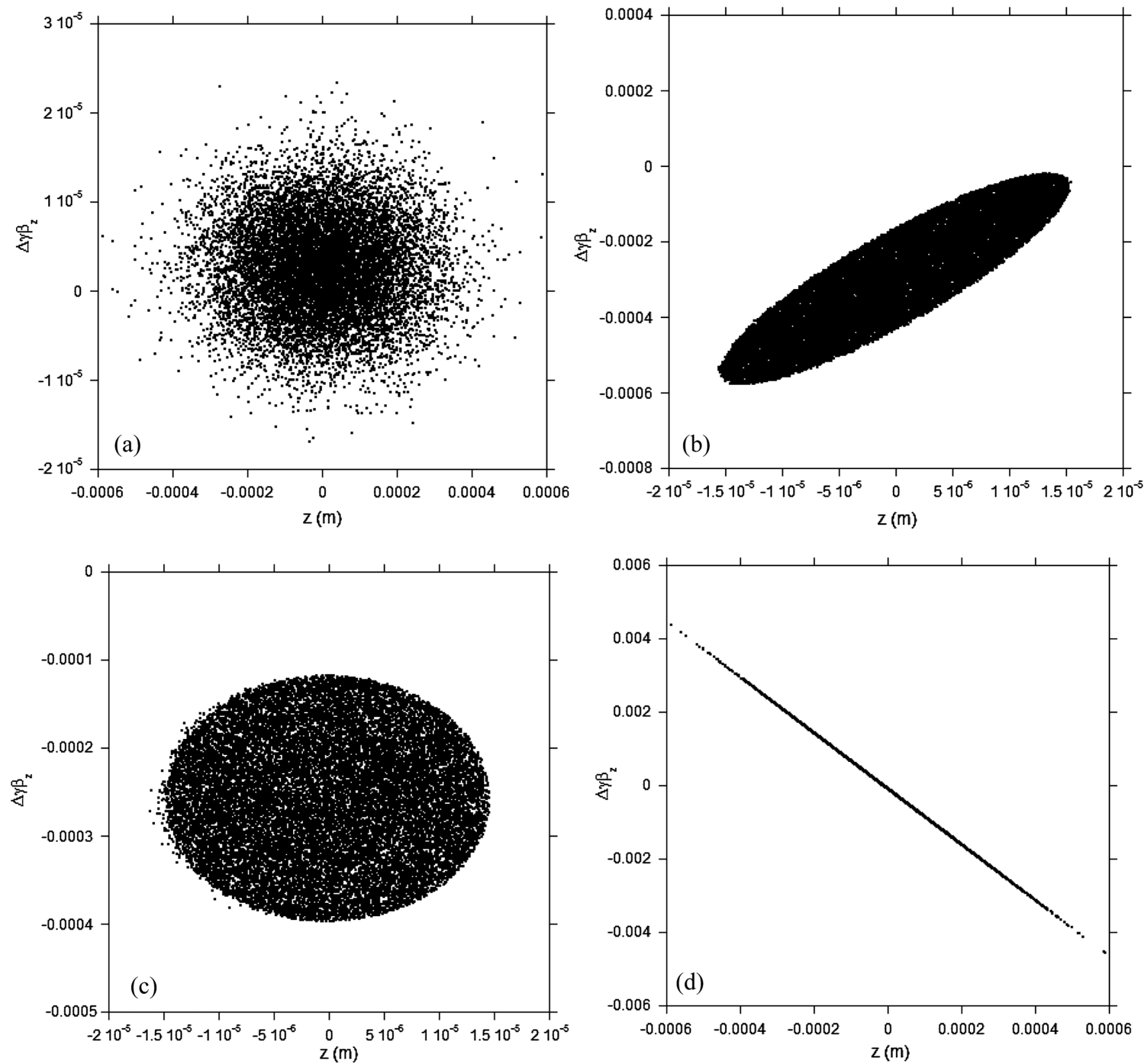

FIG. 6. Comparison of the initial longitudinal phase space (a) (the same for all three cases) and the final longitudinal phase spaces, for very low bunch charges, (b) case C, (c) case D, and (d) chicane. The different appearance of the initial phase space is because it was numerically populated with a Gaussian distribution in both position and energy deviation whereas the initial transverse phase spaces (and thus the final longitudinal phase space) were populated with a 4D K-V distribution. Case D clearly shows an uncorrelated final phase space, with a few stray particles at low axial positions demonstrating the transport nonlinearities. 

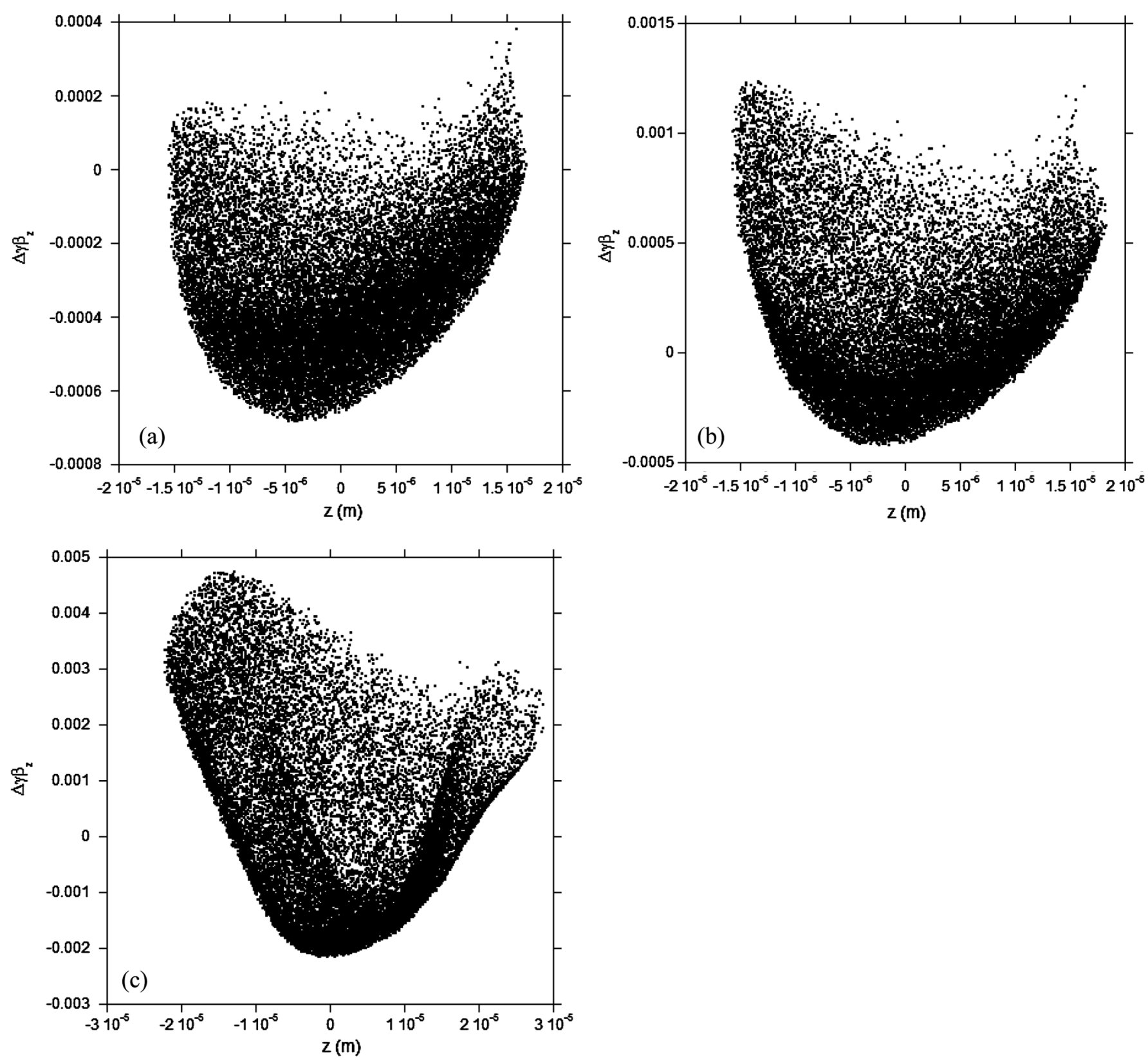

FIG. 7. (a) Final longitudinal phase for a $100 \mathrm{pC}$ bunch compressed in EEX case $\mathrm{C}-2.7 \mu \mathrm{m}$ longitudinal emittance, (b) final longitudinal phase for a $200 \mathrm{pC}$ bunch compressed in EEX case $\mathrm{C}-5 \mu \mathrm{m}$ longitudinal emittance, and (c) final longitudinal phase for a $1 \mathrm{nC}$ bunch compressed in EEX case $\mathrm{C}-33 \mu \mathrm{m}$ longitudinal emittance.

The following three sets of plots (Figs. 7-9) show the deformation in final longitudinal phase space arising from CSR forces, for case C, case D, and the chicane, each with bunch charges of 100, 200, and $1 \mathrm{nC}$.

Note, in comparing Figs. 7-9, there is no initial energy slew required for the EEX compression. The coupling between the CSR wake and final axial position when using a chicane is clearly seen in Fig. 9, as the final distribution gets folded over in longitudinal position. The longitudinal emittance growth in the chicane is clearly visible as the longitudinal energy spread distorts from the CSR wake, while it is suppressed by the weaker energy-phase correlation in the EEX cases. The final longitudinal phase space plots for case D (Fig. 8) show the least structure from CSR, resulting in the lower axial emittance plotted for that case in Fig. 5(a) and the reduced sensitivity to final bunch length plotted in Fig. 5(b). The horizontal emittance growth in the EEX cases is due to the $x-z$ coupling induced in the exchange.

The slice emittance and energy spread is shown in Fig. 10 for a $1-n C$ bunch for compression in both EEX case $\mathrm{C}$ and the chicane. These simulations show another 

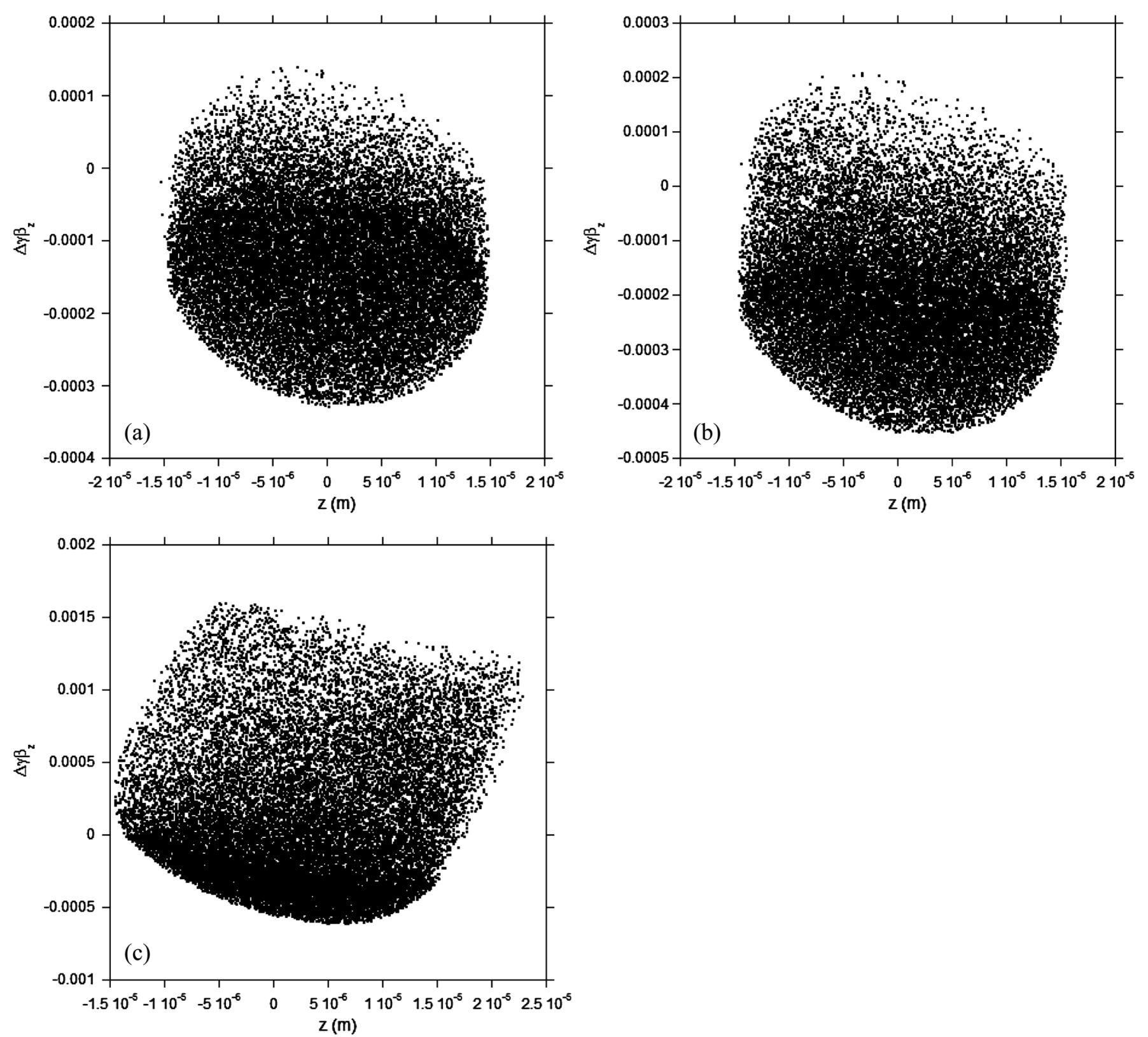

FIG. 8. (a) Final longitudinal phase for a $100 \mathrm{pC}$ bunch compressed in EEX case D-1.2 $\mu$ m longitudinal emittance, (b) final longitudinal phase for a $200 \mathrm{pC}$ bunch compressed in EEX case D-1.5 $\mu \mathrm{m}$ longitudinal emittance, and (c) final longitudinal phase for a $1 \mathrm{nC}$ bunch compressed in EEX case $\mathrm{D}-7 \mu \mathrm{m}$ longitudinal emittance.

fundamental difference between these types of compression. As commonly known, the slice emittance from compression in a chicane is significantly smaller than the rms emittance. However, the slice emittance growth from CSR with compression in an EEX is very nearly the rms emittance. The EEX is also more susceptible to slice energy spread growth than the chicane.

Next, we qualitatively consider susceptibility to the microbunch instability. Numerically, we axially modulate the initial beam distribution with a $25 \%$ harmonic current at a wavelength of about $10 \mu \mathrm{m}$. The final longitudinal phase spaces for EEX case $\mathrm{C}$ and the chicane are seen in Fig. 11, both for bunch charges of $200 \mathrm{pC}$. These plots should be compared with Figs. 7(b) and 9(b). The instability is present for both cases, but is more significant in the chicane compressor, as expected. The initial modulation leads to an enhanced energy banding in the EEX compressor, but there is no residual coupling to the final axial position (which indicates instability suppression). The emittance growth from the microbunch features, largely longitudinal for a chicane, is only horizontal for the EEX. The emittance grows from $\varepsilon_{x} / \varepsilon_{z}=9.6 / 5.3 \mu \mathrm{m}$ without seeding to $16.3 / 5.3 \mu \mathrm{m}$ with seeding for EEX case $\mathrm{C}$ and from $7.7 / 12.0 \mu \mathrm{m}$ to $7.7 / 18.6 \mu \mathrm{m}$ for the chicane case. 

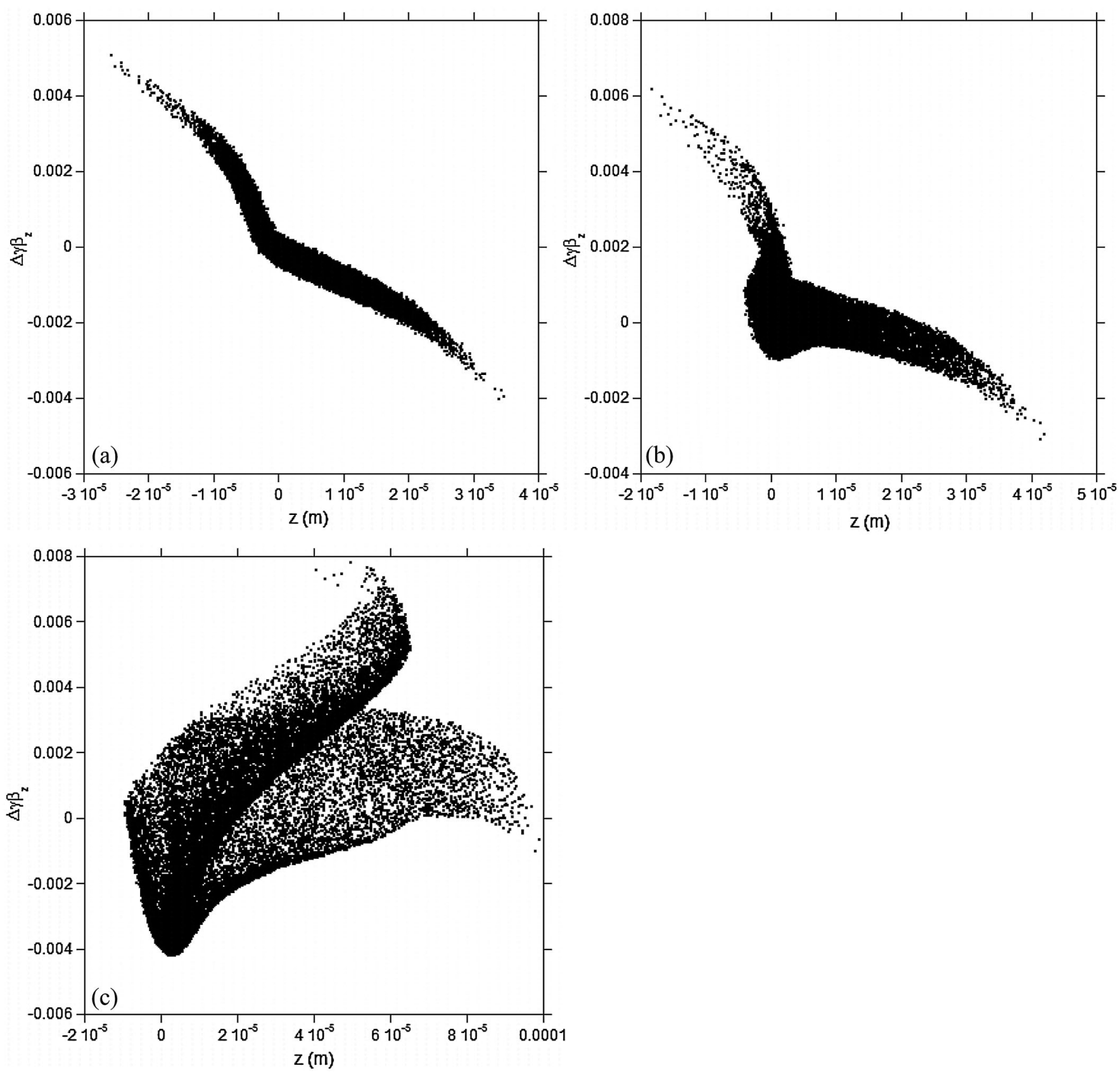

FIG. 9. (a) Final longitudinal phase for a $100 \mathrm{pC}$ bunch compressed in the chicane-6.5 $\mu$ m longitudinal emittance, (b) final longitudinal phase for a $200 \mathrm{pC}$ bunch compressed in the chicane-12 $\mu \mathrm{m}$ longitudinal emittance, and (c) final longitudinal phase for a $1 \mathrm{nC}$ bunch compressed in the chicane-77 $\mu \mathrm{m}$ longitudinal emittance.

\section{DISCUSSION}

An EEX with negative horizontal drifts between the two doglegs has significant optics advantages over the standard EEX design as initially described in [4]. These negative drifts can each be formed with six quadrupoles, as described in Appendix B, which leads to an additional complexity that might be significant for small experimental beam lines but would be acceptable for larger facilities such as linear colliders or x-ray free-electron lasers.
The main differences between compression using an EEX and a chicane arise from the novel ability of the EEX to bunch even if the momentum compaction vanishes. Compression in an EEX requires no initial beam correlations (and the beam can be maximally accelerated at the rf crest), and the final beam can be made fully longitudinally uncorrelated. The lowest emittance EEX architecture has some modest final longitudinal correlation, but even that can be made to vanish by using an alternative geometry with only minor degradation in other performance. The 

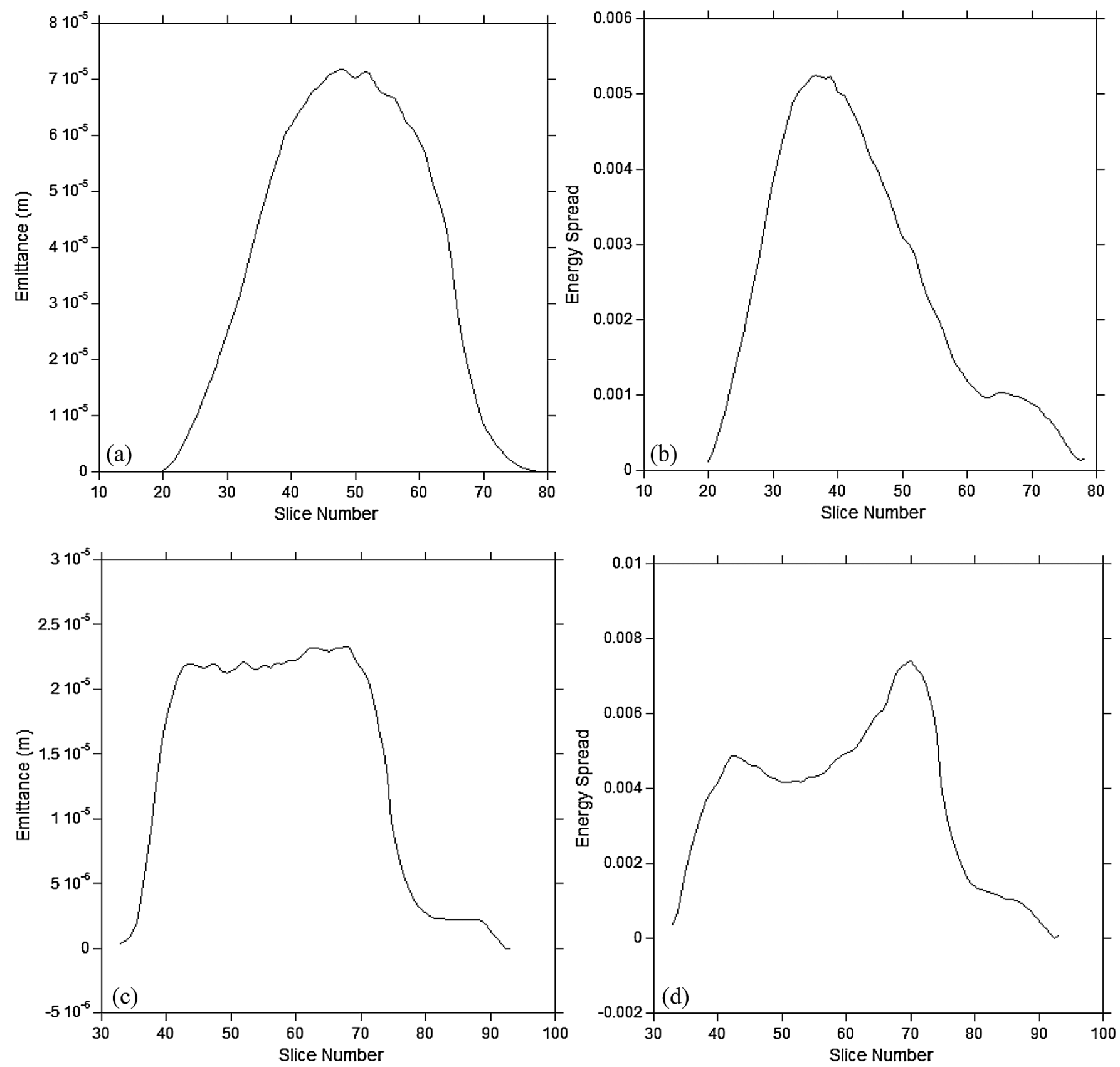

FIG. 10. (a) Slice emittance and (b) slice energy spread for the EEX for a $1 \mathrm{nC}$ bunch charge, (c) slice emittance, and (d) slice energy spread for the chicane with a $1 \mathrm{nC}$ bunch charge.

alternative geometry also has enhanced resistance to CSR effects. In addition to eliminating a potentially annoying final energy-phase correlation, another perceived advantage of using an EEX to compress the beam is its inherent microbunch instability suppression. Alternatively, one disadvantage of using an EEX for compression is that the final bunch slice transverse emittances have grown to the size of the final rms emittance, in direct contrast to compression in a chicane where the slice emittance is nearly preserved through the compression process. Another disadvantage of compressing in an EEX at high beam energy is that a long initial bunch length must be avoided to eliminate a large transverse size and geometrical emittance growth in the second dogleg of the EEX. This limitation leads to a tradeoff between electron beam energy and initial bunch length and EEX length. Thus, an EEX used for compression at high beam energy is best for converting a small initial longitudinal emittance to a small final horizontal emittance. This limitation is probably not significant, because typically two EEXs would be ganged together, where the incoming beam would have smaller transverse emittances than a longitudinal emittance and two EEXs would be 

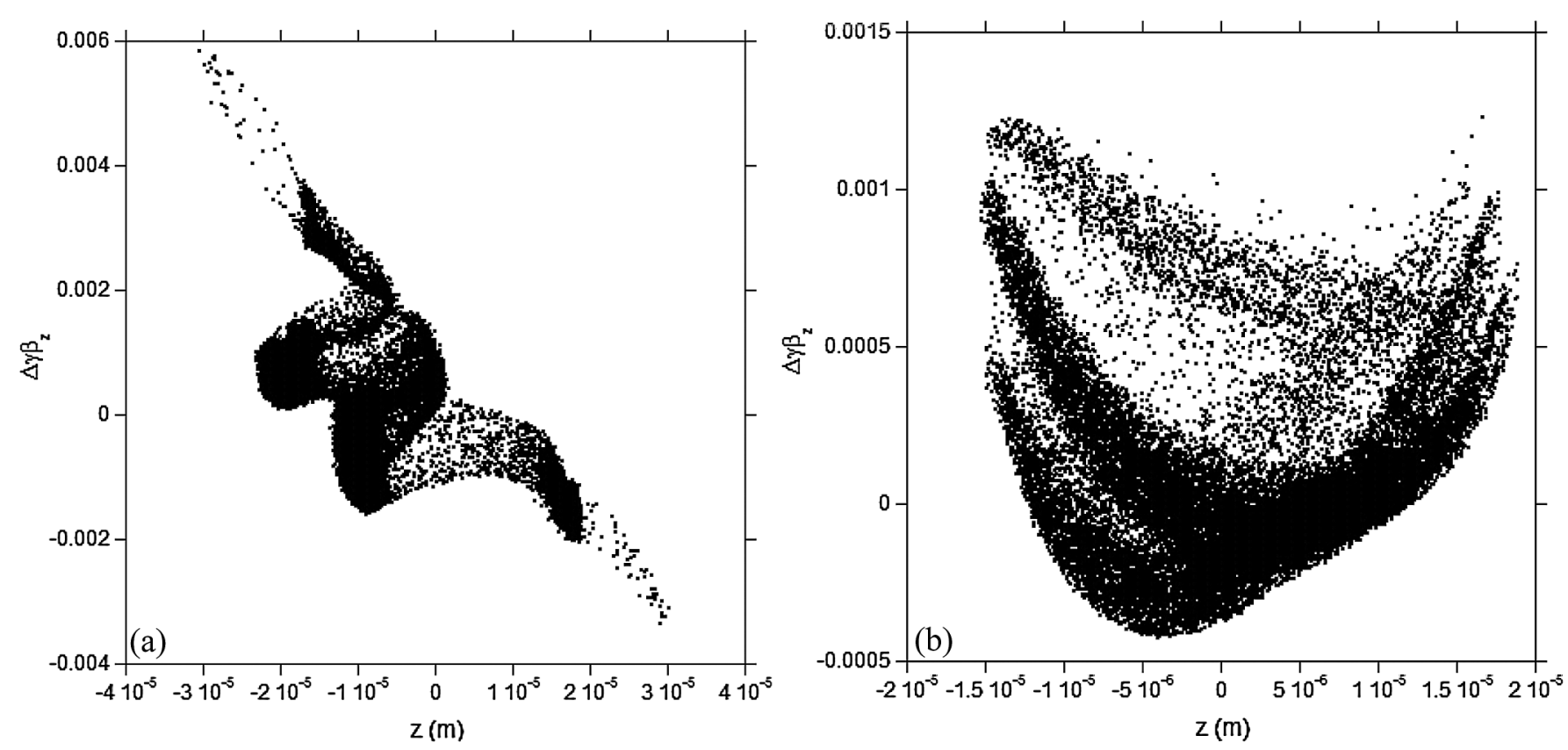

FIG. 11. Final longitudinal phase spaces showing the microbunch instability for compression in a (a) chicane and in (b) EEX case C.

needed to keep the larger emittance in the longitudinal plane. The first EEX would likely provide only modest compression, and the strong compression, in the final EEX element, would meet the requirement above. This scheme has also been proposed by Zholents [18], where the special case of using a telescope sandwiched between two identical conventional EEX structures provides the correct initial mixed transverse and longitudinal conditions for the second EEX. In any configuration, though, it is likely that the highest practical energy for using an EEX as a compressor is about $1 \mathrm{GeV}$.

Note that the emittance limitations of using an EEX result from geometrical nonlinearities in the dogleg optics. If higher-order elements can be used to linearize the dogleg optics while still allowing the transverse rf cavity to cancel the dispersion effects, this limitation can be removed. As an example, it has been shown that most of the transverse emittance growth can be removed with additional focusing and sextupoles after the EEX [26], which in principle is compatible with the two-EEX configuration described in [18].

Summarizing, bunch compression using a modified EEX design has some features significantly different than compression using a chicane, with a greater number of design parameters. Being able to compress the beam to subfemtosecond bunch lengths without requiring an initial energy-phase correlation or leaving a residual energyphase correlation might be very advantageous for certain applications. Also, compression using an EEX has less susceptibility to CSR-induced bunch length broadening and the microbunch instability which might be very advantageous for other applications. Disadvantages compared to compression in a chicane include higher transverse emittance growth from nonlinear effects, for initial bunch lengths of a picosecond or more at beam energies of $1 \mathrm{GeV}$ and higher, and the likelihood that two EEXs would have to be used instead of just one. A determination of the best architecture to use for a given beam compression requirement would require a detailed analysis comparing EEX compressor performance to chicane compressor performance.

\section{ACKNOWLEDGMENTS}

The authors are pleased to acknowledge useful discussions with Georg Hoffstaetter and his colleagues at Cornell University about EEX designs that decouple the final longitudinal phase space and with Alexander Zholents on alternative EEX schemes that can be used for bunch compression. The authors also wish to acknowledge Robert Ryne and Peter Walstrom for illuminating MARYLIE/ IMPACT simulations.

\section{APPENDIX A}

Here we show that the thin transversely deflecting rf cavity transfer matrix, Eq. (2), is fine to use, even with a collection of thick cavities, if the design is not constrained by overall length. Consider the transfer matrix from a cavity made of $n$ cells, with overall length $l$ and net coupling $k$ [27]:

$$
M_{\text {thick kicker }}=\left(\begin{array}{cccc}
1 & l & \frac{k l}{2} & 0 \\
0 & 1 & k & 0 \\
0 & 0 & 1 & 0 \\
k & \frac{k l}{2} & q k^{2} l & 1
\end{array}\right),
$$


where additionally $q=\left(1+2 n^{2}\right) /\left(12 n^{2}\right)$. The coupling from the $(4,3)$ term has been identified to degrade performance by adding a coupling emittance in quadrature to the final, swapped emittances of the form [27]

$$
\Delta \varepsilon_{\text {coupled }}^{2}=\left(\frac{1+2 n^{2}}{12 n^{2}}\right)\left(\frac{l}{\eta}\right)^{2} \sigma_{x^{\prime}}^{2}\left(\sigma_{z}^{2}-\eta^{2} \sigma_{x^{\prime}}^{2}\right),
$$

where here the beam rms values are taken at the position of the rf cavity. This term can be minimized by proper focusing into the EEX structure.

Here, alternatively, we have considered uncorrelated beams entering the modified EEX architecture and used additional optics to generate coupling to minimize other emittance growth effects, which is inconsistent with the approach described in Ref. [27]. Fortunately, we can remove the troublesome coupling elements shown in Eq. (A1) with additional optics, shown below. Alternatively, this term can be removed with a slew rf cavity (using an accelerating mode, but with the center of the beam traversing the cavity at zero phase) $[18,28]$.

Consider $n$ cells of the form shown in Eq. (A1) that are both preceded and followed by another set of $m$ cells with overall length $d$ and effective coupling $a$, both additional sets with transfer matrix

$$
M_{\text {end kicker }}=\left(\begin{array}{cccc}
1 & d & \frac{a d}{2} & 0 \\
0 & 1 & a & 0 \\
0 & 0 & 1 & 0 \\
a & \frac{a d}{2} & p a^{2} d & 1
\end{array}\right)
$$

where now $p=\left(1+2 m^{2}\right) /\left(12 m^{2}\right)$. Additionally, assume that these cavities are separated by optics with effective length $M$, with transfer matrix

$$
M_{\text {drift }}=\left(\begin{array}{cccc}
1 & M & 0 & 0 \\
0 & 1 & 0 & 0 \\
0 & 0 & 1 & 0 \\
0 & 0 & 0 & 1
\end{array}\right)
$$

The overall transfer matrix of end-kicker/drift/thickkicker/drift/end-kicker recovers the form of Eq. (2),

$$
M_{\text {net kicker }}=\left(\begin{array}{cccc}
1 & 0 & 0 & 0 \\
0 & 1 & k+2 a & 0 \\
0 & 0 & 1 & 0 \\
k+2 a & 0 & 0 & 1
\end{array}\right)
$$

if $M=-d-l / 2$ and $a=-\frac{k}{2(1-2 p)}\left[1 \pm \sqrt{1+4 \frac{l}{d} q(1-2 p)}\right]$. A negative effective drift has been key to the entire discussion of this paper, and a simple way to generate one that is suitable to realize Eq. (A5) is described in Appendix B. Also note that solution of $a$ with the positive value reduces the overall number of cells. It is likely all three cavities will have a significant number of cells, which leads to approximately $a=k \frac{3}{4}\left(\sqrt{1+\frac{4 l}{9 d}}-1\right)$. If additionally one wants all cells to have as equal a field strength as possible, an approximate solution can be found by using $a \hat{l}=k \hat{d}$ leading to $\frac{4}{3} \frac{\hat{d}}{\hat{l}}+1=\sqrt{1+\frac{4 \hat{l}}{9 \hat{d}}}$, and which can be solved with the cubic equation. The actual lengths $l$ and $d$ can be found by assuming all cells have the same length, finding integer values for $m$ and $n$ that most closely satisfy $m \hat{l}=n \hat{d}$, and peak rf field considerations.

Alternatively, if one does not want to employ negative drifts or slew rf cavities, the combination of elements can be made to look like a thin rf cavity with drifts on either side if the side rf cavities' amplitude $a$ satisfies $0=a^{2}[2 M+d(1+2 p)+l]+a k(2 M+l+d)+k^{2} l q$. This always requires $a$ to have opposite sign from $k$, and leads to requiring more rf cavities.

\section{APPENDIX B}

A negative drift optic can be constructed with either quadrupole doublets or triplets. A problem with using sequential doublets is that they both focus in the $x$ plane and, as a result, both will defocus in the $y$ plane. This could prove to be a problem in our overall optics design, so we would rather use two thin lens triplets, as shown in Fig. 12. Each triplet is defined by the following parameters:

$d_{1} \equiv$ drift before and after triplet.

$d_{2} \equiv$ drift between thin lenses.

$f_{1} \equiv$ focal length of outer lenses.

$f_{2} \equiv$ focal length of center lens.

In this scheme, if we set $f_{1}=d_{2}$ and $f_{2}=-d_{2}$ then the transfer matrix is given by

$$
M_{\text {triplet }}\left(d_{1}, d_{2}\right)=\left(\begin{array}{cccc}
-1 & -2 d_{1}+3 d_{2} & 0 & 0 \\
0 & -1 & 0 & 0 \\
0 & 0 & 1 & \left(2 d_{1}+2 d_{2}\right) / \gamma^{2} \\
0 & 0 & 0 & 1
\end{array}\right) .
$$

After placing two identical thin lens triplets sequentially in a beam line, the total transfer matrix is

$$
M_{T}\left(d_{1}, d_{2}\right)=\left(\begin{array}{cccc}
1 & -4 d_{1}+6 d_{2} & 0 & 0 \\
0 & 1 & 0 & 0 \\
0 & 0 & 1 & 4\left(d_{1}+d_{2}\right) / \gamma^{2} \\
0 & 0 & 0 & 1
\end{array}\right) .
$$

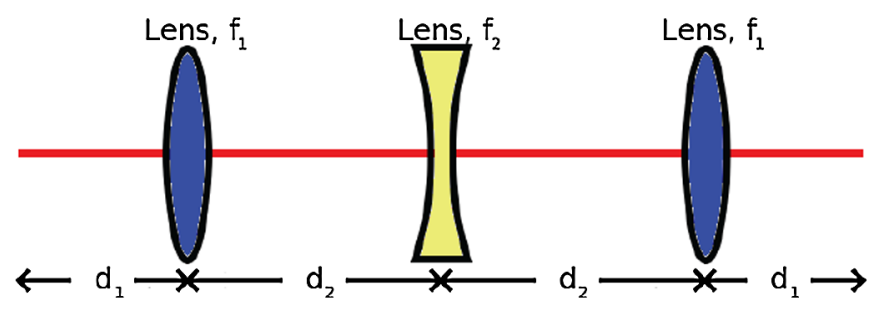

FIG. 12. Thin lens triplet definition. 


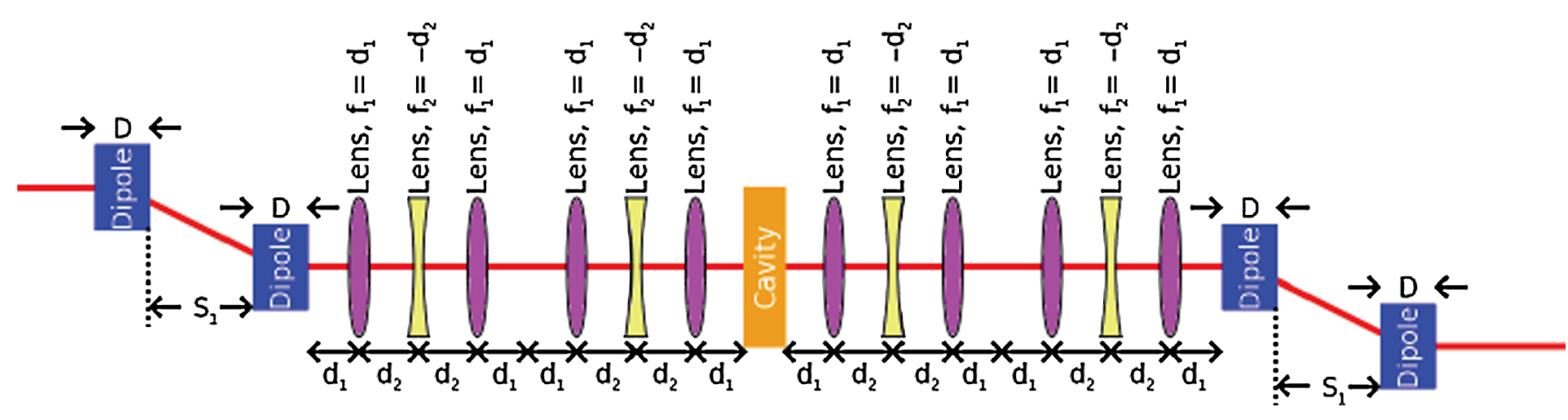

FIG. 13. Transverse-to-longitudinal emittance exchange optic with optics for negative drift lengths between the doglegs.

Appropriate choices for $d_{1}$ and $d_{2}$ will result in the proper negative value for the $M_{T, 12}$ term, and the time dispersion term vanishes at sufficiently high energy. A possible EEX configuration using this negative drift optic is thus shown in Fig. 13, where now $S_{2}$, and consequently $L$, can have any arbitrary negative value. It is important to note that this optic is not adequate to provide an equivalent negative value for $S_{1}$, for the reasons described earlier in the text.

[1] N. Yampolsky, B. Carlsten, R. Ryne, K. Bishofberger, S. Russell, and A. Dragt, arXiv:1010.1558.

[2] M. Cornacchia and P. Emma, Phys. Rev. ST Accel. Beams 5, 084001 (2002).

[3] K.-J. Kim and A. Sessler, Proceedings of the International Work Beam Cooling and Related Topics, Galena, IL, AIP Conf. Proc. No. 821 (AIP, New York, 2006), p. $115-138$.

[4] P. Emma, Z. Huang, K.-J. Kim, and P. Piot, Phys. Rev. ST Accel. Beams 9, 100702 (2006).

[5] Jinhao et al., AIP Report No. 978-0-7354-0853-1, 2010; A. Johnson, J. Ruan, H. Edwards, T. Koeth, A. Lumpkin, P. Piot, J. Santucci, Y.-E. Sun, and R. Thurman-Keup, in Proceedings of the 2010 International Particle Accelerator Conference IPAC10, Kyoto, Japan, 2010 (ICR, Kyoto, 2010) [http://accelconf.web.cern.ch/accelconf/ IPAC10].

[6] Y.-E. Sun et al., arXiv:1003.3126v1.

[7] P. Piot et al., arXiv:1007.4499v1.

[8] Steve Russell (private communication).

[9] B. E. Carlsten and S. J. Russell, Phys. Rev. E 53, R2072 (1996).

[10] K. L. F. Bane, F.-J. Decker, Y. Ding, D. Dowell, P. Emmna, J. Frisch, Z. Huang, R. Iverson, C. Limborg-Deprey, H. Loos, H.-D. Nunn, D. Ratner, G. Stupakov, J. Turner, J. Welch, and J. Wu, Phys. Rev. ST Accel. Beams 12, 030704 (2009).

[11] http://lcls.slac.stanford.edu; P. Emma, Nat. Photon. 4, 641 (2010).
[12] B. E. Carlsten and T. O. Raubenheimer, Phys. Rev. E 51, 1453 (1995).

[13] R. Li, Thomas Jefferson National Accelerator Laboratory Report No. JLAB-TN-05-046, 2005.

[14] M. Borland, Phys. Rev. ST Accel. Beams 11, 030701 (2008).

[15] B.E. Carlsten, K. A. Bishofberger, L. D. Duffy, S. J. Russell, R. D. Ryne, N. A. Yampolsky, and A. J. Dragt, Phys. Rev. ST Accel. Beams 14, 050706 (2011).

[16] B.E. Carlsten, K. A. Bishofberger, L. D. Duffy, C.E. Heath, Q. R. Marksteiner, D. C. Nguyen, R. D. Ryne, S. J. Russell, E. I. Simakov, and N. A. Yampolsky, "New X-ray Free-electron Laser Architecture for Generating High Fluxes of Longitudinally Coherent Photons" [J. Mod. Optics (to be published)], DOI 10.1080/09500340.2011.604733

[17] N. A. Yampolsky and B.E. Carlsten, "Smearing of Harmonic Current due to ISR-induced Energy Diffusion" (unpublished).

[18] A. A. Zholents and M. S. Zolotorev, Report No. Argonne National Laboratory Report No. ANL/APS/LS-327, 2011.

[19] B. E. Carlsten, K. A. Bishofberger, S. J. Russell, and N. A. Yampolsky, Los Alamos National Laboratory Report No. LA-UR-11-00485.

[20] B. E. Carlsten, L. M. Earley, F. L. Krawczyk, S. J. Russell., J. M. Potter, P. Ferguson, and S. Humphries, Phys. Rev. ST Accel. Beams 8, 062001 (2005).

[21] B. E. Carlsten, Phys. Rev. E 54, 838 (1996).

[22] E. L. Saldin et al., Nucl. Instrum. Methods Phys. Res., Sect. A 398, 373 (1997).

[23] M. Borland, Advanced Photon Source LS-287, Argonne National Laboratory (2000).

[24] P. Goldreich and D. A. Keeley, Astrophys. J. 170, 463 (1971).

[25] J. B. Murphy, S. Krinsky, and R. Gluckstern, Part. Accel. 57, 9 (1997).

[26] P. Walstrom (private communication).

[27] R. P. Fliller, III and T. W. Koeth, in Proceedings of the 23rd Particle Accelerator Conference, Vancouver, Canada, 2009 (IEEE, Piscataway, NJ, 2009), TU4PB101.

[28] N. Yampolsky (private communication). 